\title{
BPMN 2.0 Modelling for the Management of the Inspection of Execution Processes in Construction
}

\author{
Alice Gardini ${ }^{1}$, Marco Alvise Bragadin ${ }^{1}$, Berardo Naticchia ${ }^{2}$, Alessandro Carbonari ${ }^{2}$ and \\ Alessandra Corneli ${ }^{2}$
}

1 Università di Bologna, Bologna, Italy

2 Università Politecnica delle Marche, Ancona, Italy

\begin{abstract}
Nowadays digitalization is a growing challenge for the whole construction sector. Therefore, the need of supportive tools and procedures is becoming increasingly urgent in each construction project step and particularly for project supervision in the execution phase. This is a primary requirement especially for the public sector, since the legislative framework is becoming more and more focused on this aspect, in Italy as well as in the European and international context.

A formalization of the inspection procedures of project management in the construction phase is proposed through Business Process Modelling and Notation (BPMN) 2.0 language. The first key aspect of this proposal is to assume a model-based approach, which allows a more coherent information management, in contrast with the traditional document-based one. The second aspect concerns process modeling. In fact, the proposed method is based on processes, instead of BIM - based federated object-oriented models. Construction processes are modelled so that they can generate and feed the federated models themselves. In addition to this, Business Process Modelling and Notation can be used to create a collection of different procedures involved in the inspection management for construction projects. Also, BPMN model will allow an automatic feeding of an inspection management support system which will be developed in future studies, that will offer the full traceability of the procedures and the delivery of the quality certification of products. The case study of the inspection of ready-mix concrete cast-in-place process is analyzed and discussed.
\end{abstract}

(c) 2020 The Authors. Published by Budapest University of Technology and Economics \& Diamond Congress Ltd Peer-review under responsibility of the Scientific Committee of the Creative Construction Conference 2020.

Keywords: BIM, BPMN 2.0, digitalization, inspection of execution, process management

\section{Introduction}

The need of supportive procedures and tools for the digitalization of the construction field is increasing evident, especially concerning the public sector, since the legislative framework is becoming more and more focused on this aspect.

Building Information was traditionally gathered and communicated via documentation like construction drawnings, construction specifications, bill-of-quantities and written reports. Building Information Modeling (BIM) approach has shifted construction information from a document-based to a model-based system, where the complete building information is stored in a system of federated models that allows for collaborative working, for on-demand retrieval of information and digital data storage. BIM, with its centralized modelling environment, tries to answer to the need of intercommunication between the different project stakeholders, in contrast with the traditional approach of independently - developed systems and stakeholder requirements [1]. Nevertheless, despite the integration of BIM at the geometrical 
level has become widespread, it has not been the same regarding the development of transactional workflows to deal with interoperability issues. [2] [3]

The idea of adopting an approach based on System Engineering (SE) is the core concept of this paper. SE is an interdisciplinary sector that focuses on designing and managing complex systems during their whole lifecycle. In particular, SE provides languages and standards, which allow to represent the system and his operation, concentrating especially on the links between the processes and the products. [4]

Modelling of construction processes can be achieved through workflow charts. Flowcharts to represent process flows have been introduced by the Gilbreths in 1921 [5], and the current standard is ISO 5807:1985 [6], that has been widely used in the past especially in the ICT field. Flowcharting the process has different tasks, i.e. identifying inputs and outputs, identifiying the value chain, providing a visual representation of event and operationg sequences, identifying bottlenecks and controls. It is a graphical representation of a process or of a system, and many diagram techniques exist. [7]

In this contribution a formalization of the inspection procedures of project management in the construction phase is proposed using Business Process Modeling and Notation (BPMN) language. BPMN is a standard for business process modelling that is based on a flowcharting technique very similar to current flowcharts, that also provides an execution optimized business process language machine-readable [8]. Particularly, the inspection of ready-mix concrete cast-in-place process is analysed as sample case study.

\section{State of the art}

During the early 2000s, there has been a turning point for the evolution of SE field, consisted in the transition from a document-centric approach to a model-centric approach (Fig.1).
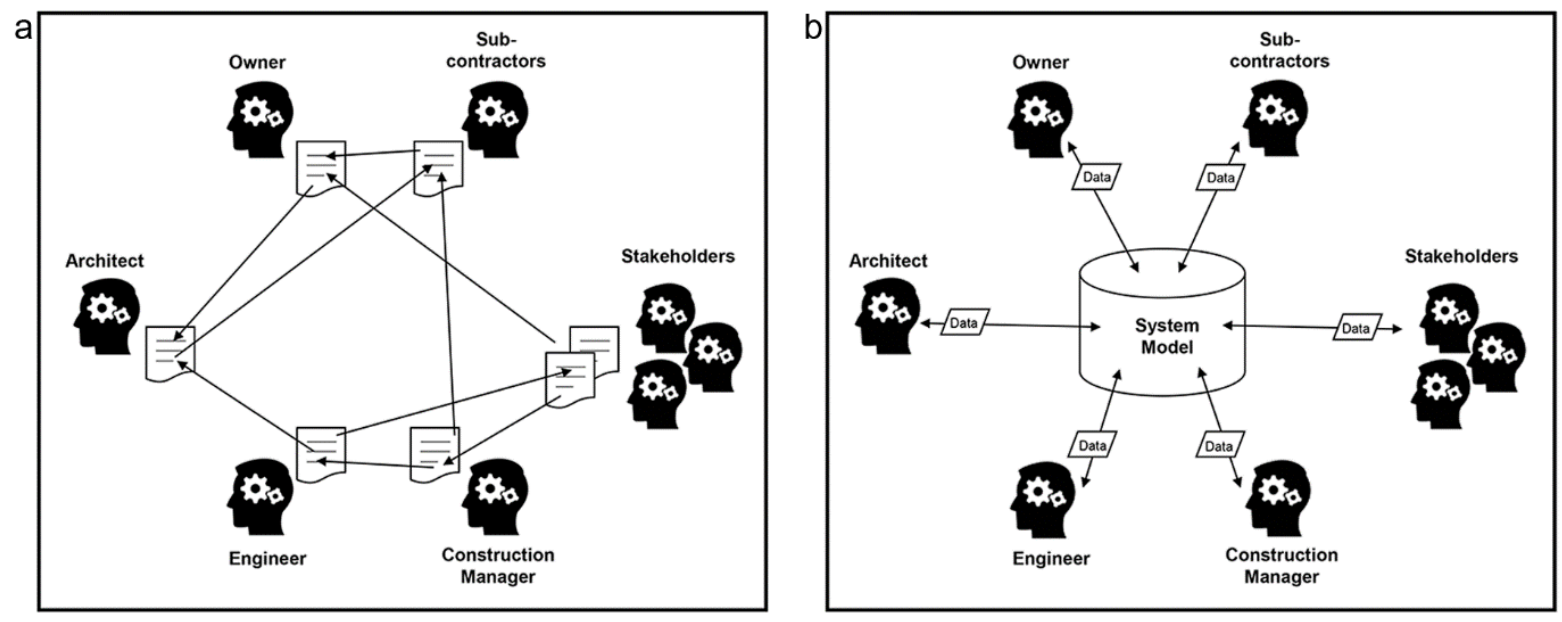

Fig. 1. (a) Document-Centric approach; (b) Model-Centric approach. [3]

However, despite the development of SE and BIM, the current practice of AEC sector is still grounded in the document-centric approach to exchange and develop building data [1]. This implicates the collection of information inside those documents, which, even if in the form of electronic files, restrict inevitably the interoperability of data exchanged between the different stakeholders. Moreover, the project data collected with this traditional method are often incomplete and inconsistent. The traditional document-centric approach describes a system literally, assuming the presence of a user between one phase and the other as a requirement, as an interpreter.

The model-centric approach responds efficiently to the lack of consistency mentioned above. In fact, many of the documents frequently required as intermediate deliverable can be generated automatically from the central model, which collects itself all the data. As Valdes et al. [8] states, "with the model-centric method, all objects depicted in different BIM or engineering tools are simply views of the underlying system model, 
they are not the model itself. In a united Model Based System Engineering MBSE-BIM model, as all modelling elements are programmatically and systemically integrated, any change that is produced will be automatically propagated to the rest of the model.".

This paper proposes to apply this Model-Based approach to the process digitalization. In this way, process models will not have documents as inputs or outputs, but they will directly condition the central model (or database). Processes are modelled in order to feed the model, in contrast with the common practice to create models first and then verify if processes are compliant with them.

\section{Proposed method}

Business Process Model and Notation (BPMN) is a modelling notation defined by Object Management Group (OMG) [9]. This notation bridges the gap between business process description flow charts and machine-readable business process descriptions [10]. Basically, this notation allows to map the visual description of a process into the appropriate execution language.

The advantages offered by BPMN are:

- An intuitive notation to represent business processes (flow charts);

- Standardization, which facilitates communication

- A comprehensible representation of constructs defined in software-execution language

Camunda Modeler [11] is the software utilized to realize the BPMN process diagrams for the research underlying this contribution. The structure of a Camunda BPMN is sketched in Fig. 2.

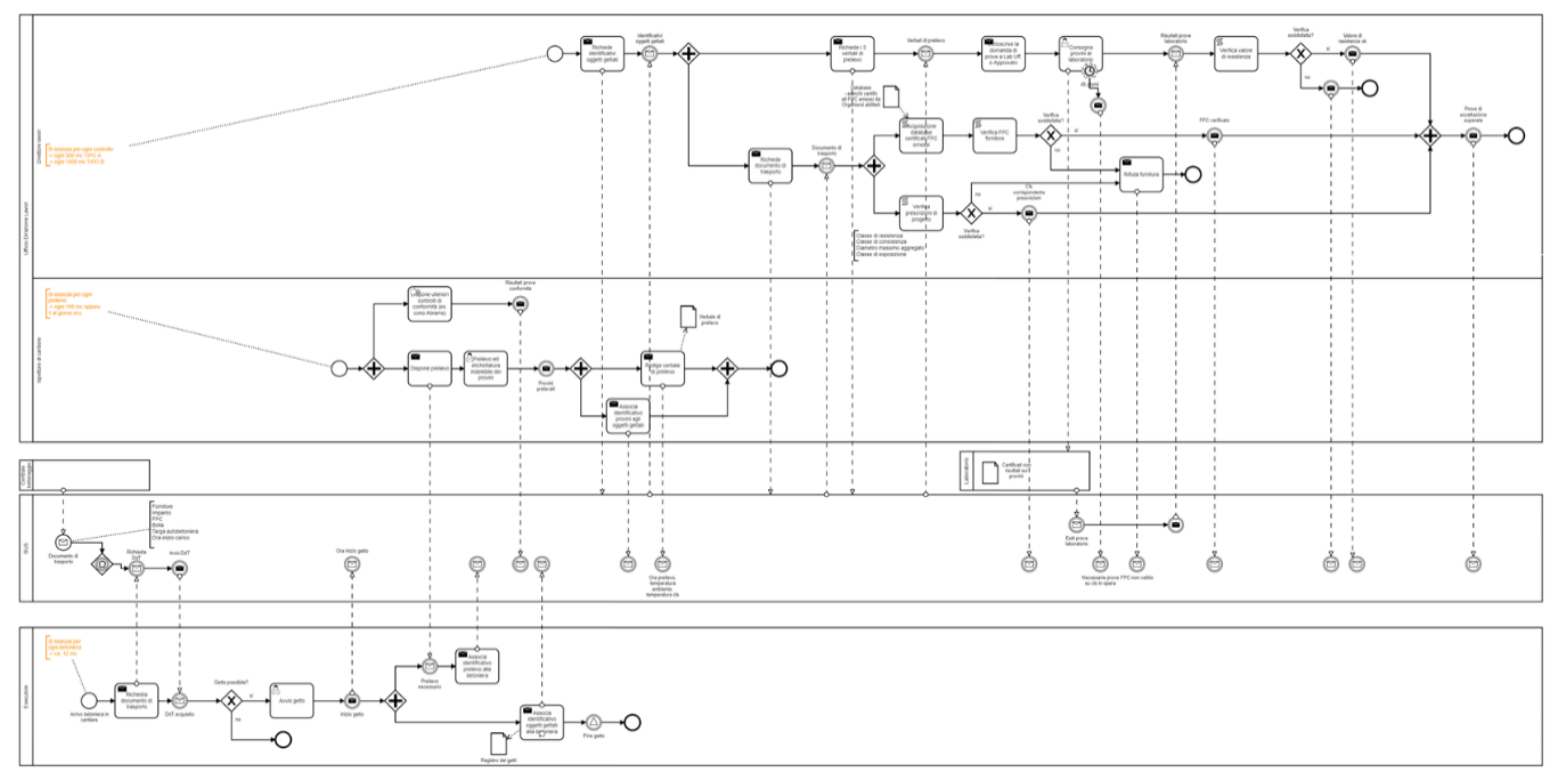

Fig. 2. Structure of a BPMN. The sample case study of inspection of ready-mix concrete cast-in-place process.

\section{Sample case study}

The inspection of ready-mix concrete cast-in-place process is analysed as sample case study. The cast-inplace of concrete in formworks is a very common construction sub-process, and the project supervisor must perform contract-based an regulation based quality tests. The inspection procedure is characterized by many correlated sub-processes, and implies a remarkable modelling complexity. Furthermore, the inspection of ready-mix concrete cast-in-place is a critical task for detecting a building structure quality and therefore needs to be assessd by several documents, following national standards, regulations and guidelines. 


\subsection{Stakeholders}

BPMN diagrams describe and highlight the processes relations between the different stakeholders involved by organizing process operations and events by lanes of the chart. Each lane corresponds to one player of the process (fig. 2).

Besides the professionals designated for the inspection and the supervision processes, it was found that another lane needs to be designed to optimize the process flow. This additional lane, defined as System BUS, is intended to act as a communication channel, which will permit to the different stakeholders to interface and exchange coherent information.

This element can be conceived as a sort of database to collect data, which will inform the different parts during all the process phases.

\subsection{Process instances}

The inspection of ready-mix concrete cast-in-place process has been modelled as a series of nested-BPMs (Fig. 3). Indeed, basing on the Italian regulations, the procedure is characterized by instances of different levels:

- $1^{\text {st }}$ level: instantiated by each inspection procedure (executed on 6 concrete sample collections)

- $\quad 2^{\text {nd }}$ level: instantiated by each concrete sample collection (executed every $100 \mathrm{mc}$ of concrete casting and at least one per day)

- $3^{\text {rd }}$ level: instantiated by each concrete casting (executed from the moment in which the truck mixer leaves the concrete mixing plant)

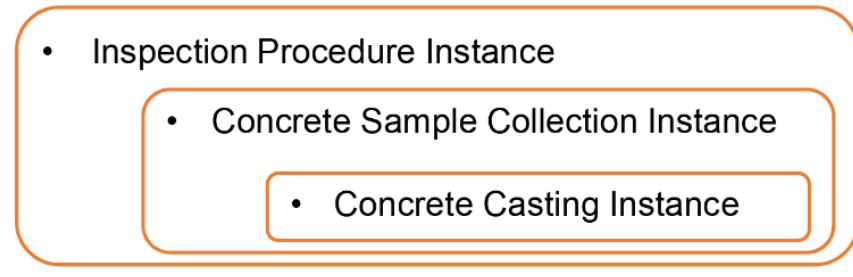

Fig. 3. Inspection of ready-mix concrete cast-in-place process: nested instances.

\subsection{Model interaction}

As mentioned above, the adoption of a model-centric approach is one of the key aspects of the modelling discussed in this paper. Particularly, the proposed BUS lane consists in a database aimed at collecting all the data directly from each stakeholder. In this way the information could be stored in a coherent, even automatic way. Consequently, the different stakeholders could just query the database to obtain the information.

Additionally, the proposed method does not ask the stakeholders to manipulate the federated model themselves. Otherwise, the instances are modelled in order to feed the database, which will inform the model itself.

Considering the sample case study, the following steps are fundamental for the interaction with the model:

- Concrete mixer ID - Concrete sample collection ID association

- Concrete mixer ID - Cast Object ID association

- Concrete sample collection ID - Cast Object ID association

Thanks to these bijective relations, once an inspection procedure is completed, it would be possible to automatically inform the model objects, for example by adding a Boolean field to the IFC object structure intended to record the result of the inspection (fig. 4). 
a

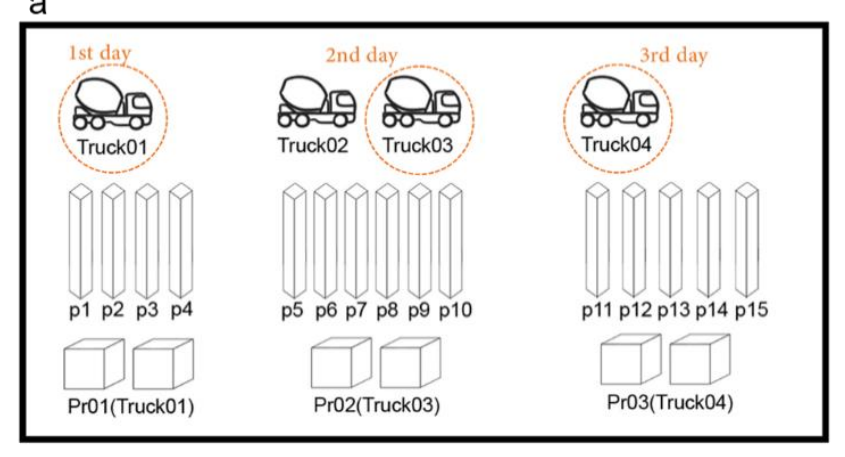

$\mathrm{b}$

\begin{tabular}{|c|c|c|c|}
\hline Object & Casting & Sample & Inspection \\
\hline p1 & Truck01 & Pr1(Truck01) & Contr1(Pr1, $\operatorname{Pr} 2, \operatorname{Pr} 3)$ \\
\hline p2 & Truck01 & Pr1(Truck01) & Contr1(Pr1,Pr2,Pr3) \\
\hline p3 & Truck01 & Pr1(Truck01) & Contr1(Pr1,Pr2,Pr3) \\
\hline p4 & Truck01 & Pr1(Truck01) & Contr1(Pr1,Pr2,Pr3) \\
\hline P5 & Truck02 & Pr2(Truck03) & Contr1(Pr1,Pr2,Pr3) \\
\hline P6 & Truck02 & Pr2(Truck03) & Contr1(Pr1,Pr2,Pr3) \\
\hline P7 & Truck02 & Pr2(Truck03) & Contr1(Pr1,Pr2,Pr3) \\
\hline P8 & Truck03 & Pr2(Truck03) & Contr1(Pr1,Pr2,Pr3) \\
\hline P9 & Truck03 & Pr2(Truck03) & Contr1(Pr1,Pr2,Pr3) \\
\hline P10 & Truck03 & $\operatorname{Pr} 2$ (Truck03) & Contr1(Pr1,Pr2,Pr3) \\
\hline P11 & Truck04 & Pr3(Truck04) & Contr1(Pr1,Pr2,Pr3) \\
\hline P12 & Truck04 & Pr3(Truck04) & Contr1(Pr1,Pr2,Pr3) \\
\hline P13 & Truck04 & Pr3(Truck04) & Contr1(Pr1, $\operatorname{Pr} 2, \operatorname{Pr} 3)$ \\
\hline P14 & Truck04 & Pr3(Truck04) & Contr1(Pr1,Pr2,Pr3) \\
\hline p15 & Truck04 & Pr3(Truck04) & Contr1(Pr1,Pr2,Pr3) \\
\hline
\end{tabular}

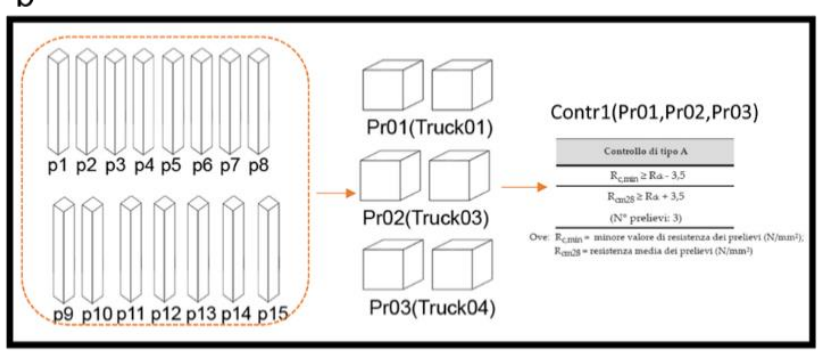

Fig. 4. Example of inspection of ready-mix concrete cast-in-place process: (a) sample collecting instance, (b) inspection procedure instance, and (c) database.

\section{Results and conclusions}

The research work has explored the potentiality of applying BPMN to the AEC sector with a positive ouput. In the same way, more BPMN based procedures concerning the management of the inspection of construction execution processes could be modelled in future studies, in order to create a collection of procedures ready to use by researchers and professionals.

The present contribution has permitted to outline the architecture of a product management system that could be the basis for the development of a tool to support the different stakeholders of building and construction projects, particularly in the execution phase. The use of BPMN - based support tools could guarantee a total traceability of the procedures, facilitating the supervision and preventing the risk of irregularities and quality non-conformities, often ecountered in the concrete cast-in-place sub-processes. Also, future research work could be aimed at developing a process-oriented digital quality assessment procedure for construction products and deliverable built with BPMN.

\section{Acknowledgements}

Research grant PRIN 2017 "A distributed Digital collaboration framework for small and medium-sized engineering and construction enterprises".

\section{References}

[1] F. Opitz, R. Windisch, R.J. Scherer R.J. Integration of document - and model - based building information for project management support. Proceedings of the Creative Construction Conference 2014, CC2014. Procedia Engineering 85, pp. 403-411. https://doi.org/10.1016/j.proeng.2014.10.566

[2] S. Azhar. Building information modeling (BIM): Trends, benefits, risks, and challenges for the AEC industry. Leadership and management in engineering, Vol. 11, no. 3, 241-252, 2011. https://doi.org/10.1061/(asce)lm.1943-5630.0000127

[3] J. Laitinen. Model Based Construction Process Management. Durability of building materials and Components 8 . National research council Canada, 1999.

[4] L. Delligatti. SysML distilled: A brief guide to the systems modeling language. 2013, Addison-Wesley.

[5] F.B. Gilbreth, L.M. Gilbreth. Process Charts. American Society of Mechanical Engineers (ASME) 1921 U.S.

[6] International Standard Organization. ISO 5807:1985 Information Processing - Documentation symbols and conventions for data, program and system flowcharts, program network charts and system resources charts. 1985 ISO.

[7] M.A. Fryman. Quality and process improvement. 2001, Delmar Pub. ISBN: 978-0766828728.

[8] F. Valdes, R. Gentry, C. Eastman, S. Forrest. Applying systems modeling approaches to building construction. In ISARC. Proceedings of the International Symposium on Automation and Robotics in Construction. Vol. 33, p. 1. 2016. IAARC Publications. https://doi.org/10.22260/isarc2016/0102

[9] Object Management Group. Business Process Model and Notation (BPMN) version 2.0. 2011.

[10] P. Y. H. Wong, J. Gibbons. A Process Semantics for BPMN. In: S. Liu, T. Maibaum, K. Araki (eds). Formal Methods and Software Engineering. ICFEM 2008. Lecture Notes in Computer Science, vol 5256. Springer, Berlin, Heidelberg. https://doi.org/10.1007/978-3540-88194-0_22

[11] Camunda. Camunda modeler. www.camunda.com (accessed december 2019). 